\title{
Cervical scar satisfaction post conventional thyroidectomy
}

\author{
Cherian Felix ${ }^{1}$, Jonathon O. Russell ${ }^{2}$, Solaiman Juman ${ }^{1}$, Steve Medford ${ }^{3}$ \\ ${ }^{1}$ The University of the West Indies, Department of Otolaryngology, Head and Neck Surgery, St. Augustine, Trinidad; ${ }^{2}$ Johns Hopkins Medicine, \\ Division of Head and Neck and Endocrine Surgery, Department of Otolaryngology, Head and Neck Surgery, Baltimore, MD, USA; ${ }^{3}$ Department of \\ Otolaryngology, Head and Neck Surgery, San Fernando General Hospital, San Fernando, Trinidad \\ Contributions: (I) Conception and design: C Felix; (II) Administrative support: S Medford, S Juman; (III) Provision of study materials or patients: C \\ Felix, S Medford; (IV) Collection and assembly of data: C Felix; (V) Data analysis and interpretation: C Felix; (VI) Manuscript writing: All authors; \\ (VII) Final approval of manuscript: All authors. \\ Correspondence to: Dr. Jonathon O. Russell. 601 N Caroline St., $6^{\text {th }}$ Floor, Baltimore, MD 21287, USA. Email: jrusse41@jhmi.edu.
}

Background: Thyroidectomy is a commonly performed procedure with large centres performing thousands of thyroid surgeries per year. The traditional Kocher mid-cervical incision has been the standard approach to thyroidectomy since the late 1870 s with predictable results. The introduction of minimally invasive and remote access techniques seeks to reduce the morbidity of a mid-cervical scar incision.

Methods: Ninety-five [95] patients who underwent this approach over the thirty [30] months period from January 2015 to July 2017 were administered the validated Patient Scar Assessment Questionnaire and the collected data analysed.

Results: Forty-eight [48] patients, (50.5\%) met inclusion criteria and provided responses with a mean time since surgery of 18.9 months. There is a high level of satisfaction with $91.7 \%$ of patients in the study having scores consistent with scar satisfaction after conventional thyroidectomy. While patients were satisfied with the appearance of their scars, a majority of patients $(n=28,58.3 \%)$ were at least slightly conscious of their incisions, with more than $10 \%$ of patients $(n=5)$ reporting significant levels of self-consciousness. Patients of Mixed race were found to have slightly elevated scores on PSAQ compared with IndoTrinidadian and AfroTrinidadian races $(\mathrm{P}=0.002$ and $\mathrm{P}=0.006)$.

Conclusions: Most patients were satisfied with their scar results in the years after thyroidectomy. Despite the high level of satisfaction, a majority of patients are self-conscious about the presence of a mid-cervical incision. Further research is needed to elucidate causes and the effects of this finding.

Keywords: Thyroidectomy; remote access thyroidectomy; scar; patient satisfaction; patient consciousness; appearance

Submitted Jul 18, 2019. Accepted for publication Nov 04, 2019.

doi: 10.21037 /gs.2019.11.17

View this article at: http://dx.doi.org/10.21037/gs.2019.11.17

\section{Introduction}

The conventional cervical incision, or Kocher incision, has been the traditional approach to thyroidectomy since it was first introduced by Theodore Kocher in the latter part of the $19^{\text {th }}$ century (1). He mainly used the collar or mid cervical incision, as he recognized that this approach gave the best cosmetic results.

Since the development of minimally invasive techniques, there has been a drive to apply these approaches in almost all specialties of surgery (2). Thyroid surgery has not been an exception to this, with the results of decreased hospital stay, post-operative pain, and improved patient satisfaction (3-5).

Even though there is increasing utilization of these techniques around the world, these facilities are not readily available in the Caribbean setting, and the Kocher incision remains the mainstay of surgical approach to the thyroid gland (6-8).

The purpose of this study was to determine if patients 
are satisfied with their surgical scar after conventional thyroid surgery incision.

\section{Methods}

\section{Patient sample}

Approval for this study was obtained from the BioEthics Committee of the South West Regional Health Authority (CCE0008). The study sample included patients who underwent a total or hemithyroidectomy at the San Fernando General Hospital, Trinidad, by the Otolaryngology and Head and Neck Surgical Team. Ninety-five [95] patients were identified by review of operative records from the time period January 2015 to July 2017.

Exclusion criteria were patients who were under the age of 18 , underwent additional procedures during surgery requiring extension of the incision, complications of surgery requiring reopening of the incision, wound infection, treatment for hypertrophic or keloid scar formation with steroid injections, and reoperation after initial surgery.

Patients were contacted via telephone and verbal consent was obtained before administration of the validated Patient Scar Assessment Questionnaire (PSAQ) was done by a standardized protocol. Forty-eight [48] patients were contacted and agreed to complete the questionnaire.

\section{Survey instrument}

The survey instrument was divided into two [2] sections A and B. Section A; included patient demographic and surgery related features: sex, age, race, time elapsed since surgery, type of surgery and histopathology report. This information was extracted from review of the patients' medical records.

The second part (Section B) of the survey instrument, contained the questions pertaining to patient satisfaction and scar consciousness. The PSAQ is a validated patient scar assessment tool (9). It consists of five (5) domains or subscales: Appearance, Symptoms, Scar Consciousness, Satisfaction with Appearance and Satisfaction with Symptoms. Of note, the Symptoms subscale is the only domain not yet validated by the instrument creators as applicable to linear scars.

The PSAQ was designed in such a way as to allow each domain to operate independently of the others and therefore the use of only 2 of the 5 subscales can be employed without affecting overall validity. Three of the five subscales were utilized in order to reduce the duration of the interview and to increase patient participation. These were: Consciousness, Satisfaction with Appearance and Satisfaction with Symptom scales.

The Consciousness subscale consists of 6 questions, the Satisfaction with Appearance and Satisfaction with Symptoms consists of 8 and 5 questions respectively. Patient responses are scored on a Likert type scale with a point range of one to four. A score of one corresponded to an answer of patient being very satisfied and four being very dissatisfied. Scores were summed to give a total score for Consciousness of 24 (range, 6 to 24), Satisfaction with Appearance 32 (range, 8 to 32) and Satisfaction with Symptoms 20, (range, 5 to 20). A total maximum overall score of 76 was possible (range, 19 to 76 ). Higher scores therefore corresponded to decreased patient satisfaction with cosmetic result.

\section{Surgical technique}

The conventional thyroidectomy incision was made in a skin crease typically placed $2 \mathrm{~cm}$ above the sternal notch. A standardized skin closure using subcuticular interrupted vicryl sutures was used for patients included in the study. Passive drains were used for all patients and these were inserted through the surgical incision site.

\section{Statistical analysis}

Data analysis was conducted using SPSS version 25 software. One-way analysis of variance (ANOVA) and independent sample $t$-test were utilized to determine if demographic or surgery related features were significant factors in predicting patient satisfaction with scar outcome.

\section{Results}

\section{Patient characteristics}

Of the 48 survey participants, 44 (91.7\%) were female and $4(8.3 \%)$ were males. The mean patient age at the time of surgery was $51.08( \pm 10.98)$ years with the youngest participant being 29 years old and the oldest 72 years. The ethnic distribution of our patients was mainly IndoTrinidadian-27 patients (56\%) followed by AfroTrinidadian 14 patients (29\%), and 7 patients (15\%) identified themselves as being Mixed Race.

The median time since surgery was 18.5 months. 
Table 1 Summary of patient characteristics, demographics and surgery related features after conventional cervical thyroidectomy surgery

\begin{tabular}{lc}
\hline Characteristics & Total sample \% [n] \\
\hline Number of patients & 48 \\
Age at time of surgery & $2.1 \%[1]$ \\
$0-29$ & $37.5 \%[18]$ \\
$30-49$ & $54.2 \%[26]$ \\
$50-69$ & $6.2 \%[3]$ \\
$>70$ & \\
Sex & \\
Male & $8.3 \%[4]$ \\
Female & $91.7 \%[44]$ \\
Ethnicity & \\
IndoTrinidadian & \\
AfroTrinidadian & $56.3 \%[27]$ \\
Mixed & $29.2 \%[14]$ \\
Type of surgery & $14.6 \%[7]$ \\
Total thyroidectomy & \\
Right hemithyroidectomy & \\
Left hemithyroidectomy & \\
Histologic diagnosis & $26.3 \%[10]$ \\
Benign & $72.9 \%[35]$ \\
Malignant & \\
\hline Unavailable & \\
\hline
\end{tabular}

The study sample included 35 patients $(72.9 \%)$ who underwent total thyroidectomy and $13(27.1 \%)$ who had hemithyroidectomy performed. Twenty-eight surgical specimens were diagnosed histologically as benign thyroid disease and 10 were diagnosed as malignant. The validated results for 10 surgical specimens were not available. This data is summarized in Table 1.

\section{Results of PSAQ}

The mean scores for patients in the Consciousness domain was $7.8 \pm 2.7$ (mean $\pm \mathrm{SD}$ ), in the Satisfaction with Appearance $10.4 \pm 3.0$ and Satisfaction with Symptoms 5.9 \pm 1.7. While overall the scores were relatively low, 5 patients $(10 \%)$ of the study population had elevated scores

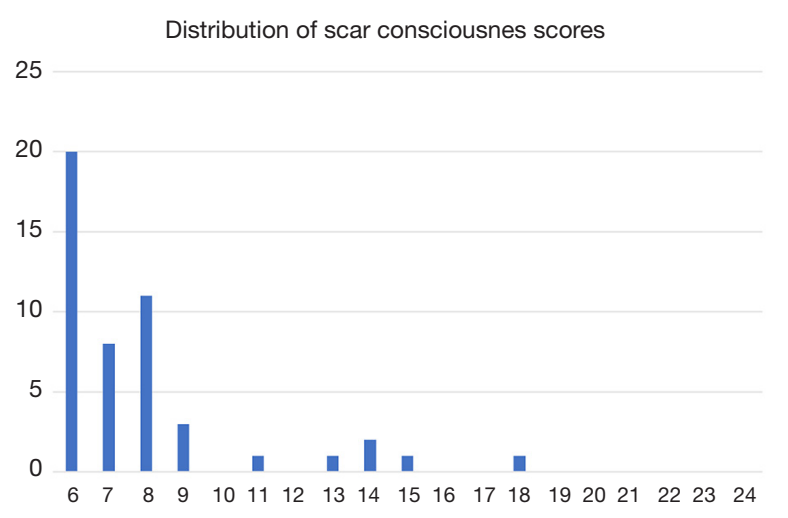

Figure 1 Distribution of PSAQ Consciousness subscale of patient scores. Minimum score achievable 6 (not; maximum score 24; $x$-axis frequency, $y$-axis score.

in the Consciousness subscale that indicated that there was a significant degree of self-consciousness with regards to the presence of a neck scar (Figure 1).

The mean total PSAQ score was $24.43 \pm 6.4$, out of a total maximum score of 76 , and scores ranged from 19 to 41 . A score of 38 or greater was considered indicative of patient dissatisfaction with scar outcome. Based on this cut-off point, two patients were deemed as having dissatisfaction with surgical scar (Table 2). The distribution of patient PSAQ scores is seen in Figure 2.

\section{Results of ANOVA and independent t-test}

In the evaluation of patient race, ANOVA analysis detected a significant difference in the overall PSAQ scores of patients of Mixed Race having an average score that was higher compared to patients of IndoTrinidadian and AfroTrinidadian race, $\mathrm{P}=0.006$ and $\mathrm{P}=0.002$ respectively.

However, ANOVA and independent $t$-test analysis did not detect any significant difference in patient scores based on patient age, gender, time elapsed since surgery, histology of specimen, or type of surgery performed.

\section{Discussion}

Incisions associated with thyroid surgery have long been perceived as a source for cosmetic concern amongst surgeons because of its location of the prominent anterior aspect of the neck. This has fuelled the drive towards minimally invasive thyroid surgery, robotic and laparoscopic associated techniques, and scarless thyroid surgery (10). 
Table 2 Characteristics of patients found to have dissatisfaction with surgical scar based on elevated Total PSAQ score greater than 38

\begin{tabular}{lllccccccc}
\hline Age & Gender & Race & Time post Op/mths & Type of Sx & Histology & CS & SAS & SSS & Total PASQ score \\
\hline 57 & Female & IndoT & 26 & TT & Benign & 15 & 19 & 7 & 41 \\
51 & Female & IndoT & 36 & LH & Benign & 18 & 16 & 6 & 40 \\
\hline
\end{tabular}

IndoT, IndoTrinidadian; mths, months; TT, total thyroidectomy; LT, left hemithyroidectomy; CS, consciousness score; SAS, Satisfaction with Appearance Score; SSS, Satisfaction with Symptoms score; PSAQ, Patient Scar Assessment Questionnaire.

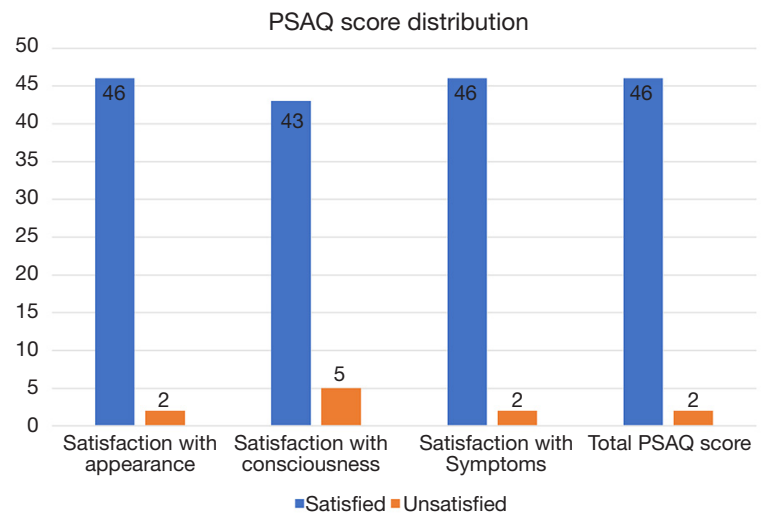

Figure 2 Satisfaction with Appearance Distribution, Scar Consciousness Score Distribution, Satisfaction with Symptoms Score Distribution and Total PSAQ Score Distribution. The $\mathrm{x}$-axis shows the number of patients and the y-axis shows the subscale categories.

However, there remains much controversy with regards to evidence to support that this view point is shared by patients who undergo thyroidectomy.

Our study assessed the satisfaction of patients post conventional cervical thyroidectomy. The findings of this study corroborate those of others which found that overall patient scar satisfaction after conventional thyroidectomy incision is very high (11-13). In our study it was observed that only two patients were found to have scores that indicated that there was a significant level of dissatisfaction with surgical scar. Notably, their scores were only marginally elevated.

Of note, there were five patients who indicated from their scores in the consciousness subscale that they were self-conscious about their scars. This showed there is a subgroup of patients who although they did not objectively identify any problems with scar symptoms or appearance, were still conscious of having a scar on their necks.

A significant difference in patient scar satisfaction was demonstrated based on the race of patients. Patients who identified themselves as being of Mixed Race consistently gave responses to the PSAQ that were statistically significantly higher compared to other racial groups. It is unclear if these higher scores were due to an increased predisposition to the formation of hypertrophic scar or wound healing idiosyncrasies.

Multiple other studies have demonstrated difference in scar satisfaction based on patient gender, with female patients generally having a greater degree of dissatisfaction compared to males $(11,14)$. This was likely not demonstrated in this study because of the small number of male participants.

The study also did not demonstrate any statistical difference in patient satisfaction based on the patient age, benign $v s$. malignant histopathology, type of operation performed (hemi or total thyroidectomy), or time elapsed since surgery.

The method used for closure of wounds for patients in this study was interrupted suture with subcuticular vicryl. Literature review of studies indicate that the method of closure chosen with regards to absorbable and nonabsorbable sutures dose not significantly impact patient perceived outcome of the scar $(15,16)$. Factors that have been shown to optimize scar outcomes are the application of cosmetic principles such as marking of patients in the upright position, avoiding elevation of subplatysmal flap to decrease postoperative neck edema and healing times, and avoidance of drain usage where possible (2).

While most patients were satisfied with their scar overall, a very high percentage of patients were at least somewhat self-conscious about their incision. This is consistent with recent reports suggesting that concerns about cosmesis are the most common of all post op adverse events associated with thyroidectomy. If a majority of patients are concerned to some degree about cosmesis, the role of "scarless" surgeries would therefore be directly related to how safe, effective, and available such procedures may be.

The benefit of minimally invasive techniques in thyroidectomy is centred around the absence of a cervical incision. Possible additional benefits of post-surgical 
recovery time and improved pain exist, but are ancillary. Because these procedures are generally more expensive, they are not always available in the Caribbean health care systems (17). Even with the consideration of factors such as decreased time of recovery and shorter periods before return to work that allow for improved overall population productivity, the cost benefit of investing in these technologies may be beyond the means of many Caribbean health providers at this time.

The role of the cervical incision on overall health is poorly defined in the literature $(3,7,12,18-21)$. It has been demonstrated that patient concerns about thyroid surgery tend to be related to concerns of the possibility of malignancy within the thyroid nodule being removed, and complications from surgery such as voice change (14). Although these above factors were beyond the scope of this study there may also be a consideration of level of education, marriage status, parity and socioeconomic factors that may play a role in patient satisfaction and perceived scar outcomes (13).

Based on a PubMed search of the literature, this is the first study of this type to assess patient satisfaction and perception of scar after thyroid surgery in Trinidad and also the English-speaking Caribbean. The study had the advantage of examining patient scar outcomes using a validated questionnaire and also of following patients over a longer period of time postoperatively.

The limitations of this study included the small sample size of only 48 patients. It is therefore to generalize obtained results. The data collected was also self-reported over telephone by patients and did not have an objective component such as scar length or observer assessment as would be considered in other scar assessment tools $(22,23)$.

\section{Conclusions}

In conclusion, this study demonstrates that there is overall a high level of satisfaction amongst patients post conventional cervical incision for thyroidectomy, despite the fact that most patients are somewhat self-conscious of their incision even more than a year after surgery. The role of remote access thyroid surgery is therefore directly related to how much more costly it is to patients, both with regards to finance, but even more with regards to complications, effectiveness and availability. Patients of mixed race were found to have a slightly elevated scores on PSAQ compared with other race groups in the study. The role of minimally invasive and scar eliminating surgical approaches in the
Caribbean should be evaluated for cost-effectiveness of application in the region.

\section{Acknowledgments}

This work was supported by Mr. Steve Medford, The University of the West Indies, St. Augustine, Trinidad.

\section{Footnote}

Conflicts of Interest: The authors have no conflicts of interest to declare.

Ethical Statement: The authors are accountable for all aspects of the work in ensuring that questions related to the accuracy or integrity of any part of the work are appropriately investigated and resolved. Approval for this study was obtained from the Bio-Ethics Committee of the South West Regional Health Authority (CCE0008). Patients were contacted via telephone and verbal consent was obtained before administration of the validated Patient Scar Assessment Questionnaire (PSAQ) was done by a standardized protocol.

\section{References}

1. Linos D, Chung WY, editors. Minimally invasive thyroidectomy. Berlin: Springer, 2012:257.

2. Terris DJ, Seybt MW, Elchoufi M, et al. Cosmetic Thyroid Surgery: Defining the Essential Principles. Laryngoscope 2007;117:1168-72.

3. Miccoli P, Berti P, Raffaelli M, et al. Comparison between minimally invasive video-assisted thyroidectomy and conventional thyroidectomy: A prospective randomized study. Surgery 2001;130:1039-43.

4. Zachariah SK. Gas-less Video-assisted Thyroidectomy for a Solitary Thyroid Nodule: Technical Report of the First Case Performed at a Rural Teaching Hospital in India and Review of Literature. J Surg Tech Case Rep 2012;4:27-31.

5. Linos D. Minimally invasive thyroidectomy: A comprehensive appraisal of existing techniques. Surgery 2011;150:17-24.

6. Anuwong A, Ketwong K, Jitpratoom P, et al. Safety and Outcomes of the Transoral Endoscopic Thyroidectomy Vestibular Approach. JAMA Surg 2018;153:21-7.

7. Russell JO, Razavi CR, Garstka ME, et al. Remote-Access Thyroidectomy: A Multi-Institutional North American Experience with Transaxillary, Robotic Facelift, and 
Transoral Endoscopic Vestibular Approaches. J Am Coll Surg 2019;228:516-22.

8. Russell JO, Razavi CR, Shaear M, et al. Transoral Vestibular Thyroidectomy: Current State of Affairs and Considerations for the Future. J Clin Endocrinol Metab 2019. [Epub ahead of print].

9. Durani P, McGrouther DA, Ferguson MW. The Patient Scar Assessment Questionnaire: A Reliable and Valid Patient-Reported Outcomes Measure for Linear Scars. Plast Reconstr Surg 2009;123:1481-9.

10. Juarez MC, Ishii L, Nellis JC, et al. Objectively measuring social attention of thyroid neck scars and transoral surgery using eye tracking. Laryngoscope 2019. [Epub ahead of print].

11. Best AR, Shipchandler TZ, Cordes SR. Midcervical scar satisfaction in thyroidectomy patients. Laryngoscope 2017;127:1247-52.

12. Linos DA, Petralias A. Commentary on: A prospective comparison of patient body image after robotic thyroidectomy and conventional open thyroidectomy in patients with papillary thyroid carcinoma. Surgery 2014;156:126-7.

13. Linos D, Economopoulos KP, Kiriakopoulos A, et al. Scar perceptions after thyroid and parathyroid surgery: Comparison of minimal and conventional approaches. Surgery 2013;153:400-7.

14. Abdul-Sater L, Henry M, Majdan A, et al. What are thyroidectomy patients really concerned about? Otolaryngol Head Neck Surg 2011;144:685-90.

15. Kundra RK, Newman S, Saithna A, et al. Absorbable or non-absorbable sutures? A prospective, randomised evaluation of aesthetic outcomes in patients undergoing

Cite this article as: Felix C, Russell JO, Juman S, Medford S. Cervical scar satisfaction post conventional thyroidectomy. Gland Surg 2019;8(6):723-728. doi: 10.21037/gs.2019.11.17 elective day-case hand and wrist surgery. Ann R Coll Surg Engl 2010;92:665-7.

16. Holger JS, Wandersee SC, Hale DB. Cosmetic outcomes of facial lacerations repaired with tissue-adhesive, absorbable, and nonabsorbable sutures. Am J Emerg Med 2004;22:254-7.

17. Billmann F, Bokor-Billmann T, Voigt J, et al. Effects of a cost-effective surgical workflow on cosmesis and patient's satisfaction in open thyroid surgery. Int J Surg 2013;11:31-6.

18. Perigli G, Cortesini C, Qirici E, et al. Clinical Benefits of Minimally Invasive Techniques in Thyroid Surgery. World J Surg 2008;32:45-50.

19. Bokor T, Kiffner E, Kotrikova B, et al. Cosmesis and body image after minimally invasive or open thyroid surgery. World J Surg 2012;36:1279-85.

20. O'Connell DA, Diamond C, Seikaly H, et al. Objective and subjective scar aesthetics in minimal access vs conventional access parathyroidectomy and thyroidectomy surgical procedures: a paired cohort study. Arch Otolaryngol Head Neck Surg 2008;134:85-93.

21. Toll EC, Loizou P, Davis CR, et al. Scars and satisfaction: do smaller scars improve patient-reported outcome? Eur Arch Otorhinolaryngol 2012;269:309-13.

22. Mundy LR, Miller HC, Klassen AF, et al. Patient-Reported Outcome Instruments for Surgical and Traumatic Scars: A Systematic Review of their Development, Content, and Psychometric Validation. Aesthetic Plast Surg 2016;40:792-800.

23. Durani P, McGrouther DA, Ferguson MW. Current scales for assessing human scarring: A review. J Plast Reconstr Aesthet Surg 2009;62:713-20. 